\title{
Open Cyclic Grid Graphs Are Graceful
}

\author{
S.Venkatesh \\ Department of Basic Sciences, College of Applied Sciences, A 'Sharqiyah University, P.O. Box.42, P.C.400, \\ Sultanate of Oman
}

\begin{abstract}
In this paper, we present the graceful labeling of open cyclic grid graph and vertex cordial labeling of generalized open cyclic grid graph.

Keywords: Graph labeling, Graceful labeling, Vertex Cordial labeling

2010Mathematics Subject Classification:05C78
\end{abstract}

\section{Introduction}

Let $G(V, E)$ be any finite simple graph. For any graph theoretic notation we follow West [4]. A function $f$ is called a graceful labeling of a graph $G$ with $m$ edges, if $f$ is an injection from the vertices of $G$ to the set $\{0,1,2, \ldots, m\}$ such that when edge $u v$ is assigned the label, $|f(u)-f(v)|$ the resulting labels are distinct. In the field of graph labeling there are very few results deals with the generation of bigger graceful graphs from the smaller ones by using standard operations like join, union etc., For detailed survey refer Gallian[1]. In this direction we introduce new method of combining cycles and proved that the resulting graph is graceful. Let $f$ be a function from the vertices of $G$ to $\{0,1\}$ and for each edgexyassign the label $|f(x)-f(y)|$. Call $f$ a vertex cordial labeling of $G$ if the number of vertices(edges) labeled 0 and the number of vertices(edges) labeled 1 differs by at most 1 . The graph that admits vertex cordial labeling is called vertexcordial graph. The concept of vertexcordial labeling was introduced by Cahit [1].

A vertex $v \in V$ of a connected graph $G$ is said to be an attachment vertex, if $\operatorname{deg}(v)=2$. Let $C_{m}$ and $C_{n}$ be any cycles of length $4 k, k \geq 1$. Then $O G(1, n)=C_{m} \oplus C_{n}$ is the graph obtained by attaching a copy of $C_{n}$ with all attachment vertices of $C_{m}$. In the same way, define $O G(i, n)=O G(i-1, t) \oplus C_{n}$, that is, $O G(i, n)$ is the graph obtained by attaching a copy of $C_{n}$ with all attachment vertices of $O G(i-1, t)$.

An open cyclic grid graph is the graph $O G(1, n)\left[=C_{m} \oplus C_{n}\right]$ as defined above. Refer figure.1.An generalized open cyclic grid graph is the graph $O G(i, n), i \geq 2$.

In this paper, for $n \equiv 0(\bmod 4)$ we prove that the open cyclic grid graph $O G(1, n)$ admits graceful labeling and generalized open cyclic grid graph $O G(i, n), i \geq 2$ admits vertex cordial labeling.

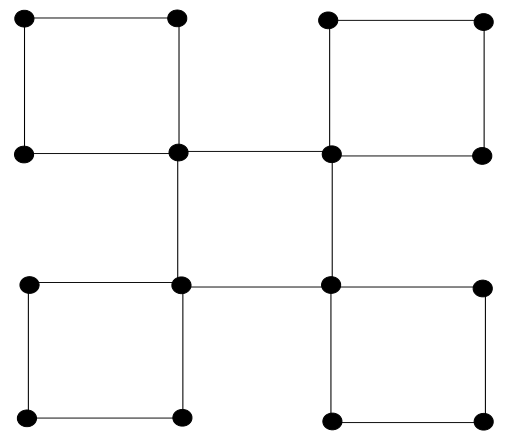

Fig.1.Open Cyclic Grid Graph $O G(1,4)=C_{4} \oplus C_{4}$

\section{Main Results}

Theorem. 2.1 Open Cyclic grid graph $O G(1, n)=C_{n_{\mathbf{0}}} \oplus \boldsymbol{C}_{\boldsymbol{n}}$ is graceful for $\boldsymbol{n}_{\mathbf{0}}, \boldsymbol{n} \equiv \mathbf{0}(\bmod 4)$.

Let $C_{n_{0}}: a_{1} e_{1} a_{2} e_{2} \ldots e_{n_{0}-1} a_{n_{0}} e_{n_{0}} a_{1}$ and $C_{n}: a_{i, 1} e_{i, 1} a_{i, 2} e_{i, 2} \ldots e_{i, n-1} a_{i, n} e_{i, n} a_{i, 1}$ be any two cycles of length $n_{0}, n \equiv 0(\bmod 4)$. Then $O G(1)=C_{n_{0}} \oplus C_{n}$ is the graph obtained as described above having $p=n_{0}+n_{0}(n-$ 1) vertices and $q=n_{0}+n_{0} n$ edges. In $O G(1)$, For $1 \leq i \leq n_{0}, i-$ even let $a_{i}=a_{i, 1}$ and $a_{i, n}=a_{i}, i-o d d$. Define the labeling $\varphi: V(O G(1, n)) \rightarrow\{0,1,2, \ldots, q\}$ as follows,

$$
\varphi\left(a_{1,1}\right)=q, \quad \varphi\left(a_{1,2}\right)=0
$$


For $3 \leq i \leq n_{0}$ and $i-o d d, \varphi\left(a_{i, 1}\right)=\varphi\left(a_{i-1, n-1}\right)-1$

$$
\varphi\left(a_{1, j}\right)= \begin{cases}\left(a_{1,1}\right)-\left(\frac{j-1}{2}\right), & j-\text { odd }, j<\frac{n}{2} \\ \left(a_{1,1}\right)-\left(\frac{j+1}{2}\right), & j-\text { odd }, j \geq \frac{n}{2} \\ \frac{j}{2}-1, & j-\text { even }\end{cases}
$$

$\varphi\left(a_{i, 2}\right)=\varphi\left(a_{i-1, n}\right)+1$,except $i=\frac{n_{0}}{2}+1$

If $i=\frac{n_{0}}{2}+1, \varphi\left(a_{i, 2}\right)=\varphi\left(a_{i-1, n}\right)+2$

For $3 \leq j \leq n, \varphi\left(a_{i, j}\right)= \begin{cases}\left(a_{i, 1}\right)-\left(\frac{j-1}{2}\right), & j-\text { odd }, j<\frac{n}{2} \\ \varphi\left(a_{i, 1}\right)-\left(\frac{j+1}{2}\right), & j-\text { odd }, j \geq \frac{n}{2} \\ \varphi\left(a_{i, 2}\right)+\frac{j}{2}-1, & j-\text { even }\end{cases}$

For $2 \leq i \leq n_{0}$ and $i-$ even, $\varphi\left(a_{i, 1}\right)=\varphi\left(a_{i-1, n-1}\right)-1$

$\varphi\left(a_{i, 2}\right)=\varphi\left(a_{i-1, n}\right)+1$, except,$i=\frac{n_{0}}{2}+1$

If $i=\frac{n_{0}}{2}+1, \varphi\left(a_{i, 2}\right)=\varphi\left(a_{i-1, n}\right)+2$

For $3 \leq j \leq n, \varphi\left(a_{i, j}\right)= \begin{cases}\varphi\left(a_{i, 1}\right)-\left(\frac{j-1}{2}\right), & j-\text { odd } \\ \varphi\left(a_{i, 1}\right)+\frac{j}{2}-1, & j-\text { even, } 4 \leq j \leq \frac{n}{2} \\ \varphi\left(a_{i, 2}\right)+\frac{j}{2}, & j-\text { even, } j>\frac{n}{2}\end{cases}$

From the above vertex labeling we observe that for $1 \leq i \leq n_{0}, 1 \leq j \leq n$ the following set $\left\{\varphi\left(a_{i, j}\right): j\right.$ odd $\}$ is a monotically decreasing sequence and for $1 \leq i \leq n_{0}$ and $2 \leq j \leq n$, the set $\left\{\varphi\left(a_{i, j}\right): j\right.$ even $\}$ is a monotically increasing sequence. From the above sequence it is clear that, for $1 \leq i \leq n_{0}$,

$$
\min \left\{\left(a_{i, j}\right): 1 \leq j \leq n, j \text { odd }\right\}>\max \left\{\left(a_{i, j}\right): 2 \leq j \leq n, j \text { even }\right\}
$$

Thus all the vertex labels are distinct and Hence $\varphi$ is injective. From the above vertex labels it is clear that all the edge values are distinct and hence the graph $O G(1, n)$ is graceful.

Conjecture 2.2.For $i \geq 2$, Generalized Open Cyclic grid graph $O G(i, n)$ is graceful.

\subsection{Illustrations to Theorem. 2.1}
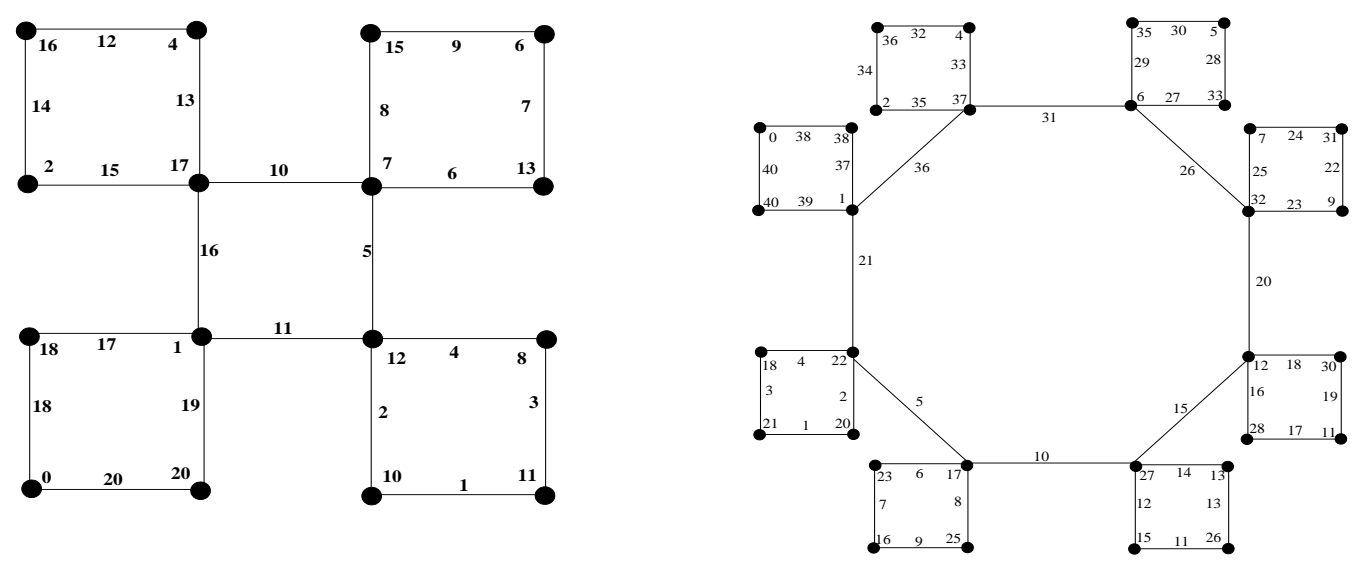

Fig.2. $O G(1)=C_{4} \oplus C_{4}$ Fig.3.OG(1) $=C_{8} \oplus C_{4}$

Theorem.2.4. Open Cyclic grid graph $O G(1, n)$ admits vertex cordial labeling for $n \equiv \mathbf{0}(\bmod 4)$.

Let $C_{m}: a_{1} a_{2} \ldots a_{m} a_{1}$ be a cycle of length $m \equiv 0(\bmod 4)$.

Let $V_{0}$ and $V_{1}$ denote the set of all vertices assigned the label 0 and 1 respectively. In the same way, let $E_{0}$ and $\mathrm{E}_{1}$ denote the set of all edges assigned the label 0 and 1 respectively.

Define the labeling $\varphi: V\left(C_{m}\right) \rightarrow\{0,1\}$ as follows,

For $1 \leq i \leq m$, let $\varphi\left(a_{i}\right)= \begin{cases}1, & \text { if } i \text { is odd } \\ 0, & \text { if } i \text { is even }\end{cases}$

From the graph $C_{m}$ it is clear that the vertices $a_{1}, a_{2}, \ldots, a_{m}$ are the attachment vertices. 
Let $C_{n}$ be acycle of length $n \equiv 0(\bmod 4)$. Consider $m$ copies of $C_{n}$ and let it be $C^{i}: b_{i, 1} b_{i, 2} \ldots b_{i, n} b_{i, 1}$ for $1 \leq i \leq n$. Now construct the graph $O G(1, n)=C_{m} \oplus C_{n}$ by merging the vertex $b_{i, 1}$ of a copy $C^{i}$ with all the attachment vertices $a_{i}$ of $C_{m}$, for $1 \leq i \leq m$.

It is observed that for $1 \leq i \leq m$, the vertex $b_{i, 1}=a_{i}$ and further the vertex $b_{i, 1}$ has the labeling either 1 or 0 . For the convenience of the labeling, arrange the edges $b_{i, j}, 1 \leq i \leq m$ and $1 \leq j \leq n$ in a sequence of the form $b_{i, 1} b_{i, 2} \ldots b_{i, n} b_{i, 1}$ where as the vertex $b_{i, 1}$ has the label either 1 or 0 .

Define the labeling $\varphi: V(\operatorname{OG}(1, n)) \rightarrow\{0,1\}$ as follows,

For $1 \leq i \leq m$ and $1 \leq j \leq n$, if the vertex $\varphi\left(b_{i, 1}\right)=1$, then $\varphi\left(b_{i, j}\right)=(1100)^{\frac{n}{4}}$ and if $\varphi\left(b_{i, 1}\right)=0$, then $\varphi\left(b_{i, j}\right)=(0011)^{\frac{n}{4}}$.

Clearly $\left|V_{0}\right|=\left|V_{1}\right|$ and $\left|E_{1}\right|=\left|E_{0}\right|$ and hence $O G(1, n)$ is vertex cordial.

In the next theorem, we prove that the generalized open cyclic grid graph admitsvertexcordial labeling. Recall thatfor $i \geq 2, O G(i, n)=O G(i-1, n) \oplus C_{n}$, is the graph obtained by attaching a copy of $C_{n}$ with all attachment vertices of $O G(i-1, n)$, where $n \equiv 0(\bmod 4)$.

Theorem.2.5.For $i \geq 2$, GeneralizedOpen Cyclic grid graph $O G(i, n)$ admits vertex cordiallabelingforn $\equiv$ $0(\bmod 4)$.

For $i \geq 2$, consider the vertex cordial graph $O G(i-1, n)$ having the attachment vertices $a_{1}, a_{2}, \ldots, a_{k}$.

Let $C_{n}$ be acycle of length $n \equiv 0(\bmod 4)$. Consider $k$ copies of $C_{n}$ and let it be $\mathrm{C}^{j}: b_{j, 1} b_{j, 2} \ldots b_{j, n} b_{j, 1}$ for $1 \leq j \leq$ $k$. Now construct the generalized open cyclic grid graph $O G(i, n)=O G(i-1, n) \oplus C_{n}$ by merging the vertex $b_{j, 1}$ of a copy $\mathrm{C}^{j}$ with all the attachment vertices $a_{j}$ of $O G(i-1, n)$, for $1 \leq j \leq k$.

The remaining part of proof follows similarly as done in Theorem. 2.4.

\subsection{Illustrations to Theorem. 2.5}
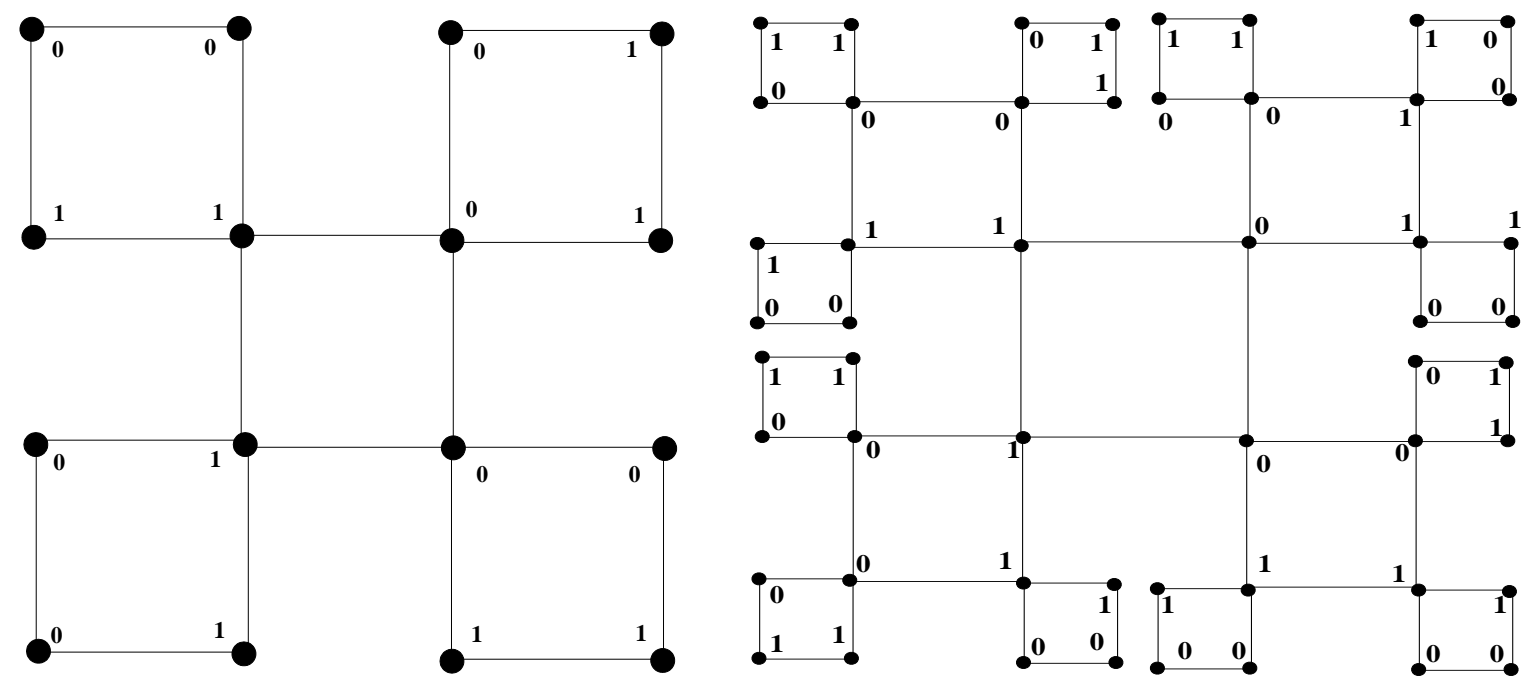

Fig.4. Vertex cordial labeling of $O G(1,4)$ Fig.5.Vertex cordial labeling of $O G(2,4)$

\section{Conclusion}

In this paper the graceful labeling of open cyclic grid graph and vertex cordial labeling of generalized open cyclic grid graph are investigated.Further it is conjectured that generalized open cyclic grid graph is graceful and it has a potential to provide motivation to investigate analogous results for different types of labeling.

\section{References:}

[1]. Cahit I, Cordial Graphs: A weaker version of graceful and harmonious Graphs, ArsCombinatoria, vol. 23, 1987. pp. $201-207$.

[2]. K.M. Koh, D.G. Rogers and T. Tan, Product of Graceful trees, Discrete Math., 31, 1980, 279 - 292, 1980.

[3]. S.Venkatesh, G.Sethuraman,Decomposition of complete graphs and complete bipartite graphs into $\alpha$-labelled trees, ArsCombin., 93 (2009) 371-385.

[4]. D.B. West. Introduction to Graph Theory, Prentice Hall of India, 2001

[5]. J.A. Gallian, A dynamic survey of Graph Labeling, The Electronic Journal Combinatorics, \#DS 6, 2013. 